\section{The Lumbar Multifidus Muscles are Affected by Medial Branch Interventions for Facet Joint Syndrome: Potential Problems and Proposal of a Pericapsular Infiltration Technique}

Low back pain is a major health problem. Like any structure of the spine and its adjacent musculature, the facet joints can be a source of pain. It has been proposed that in $15 \%-52 \%$ of patients with chronic low back pain, the facet joints are involved, but isolated facet joint pain is exceedingly rare. ${ }^{1}$ This is in accordance with the proposed biomechanical pathophysiology of facet joint syndrome. In the course of degenerative disk disease, the intervertebral disk loses height; this change leads to a segmental instability with an increased loading of the facet joints and consecutive osteoarthritic changes. To compensate for this degenerative cascade, segmental stabilization by the lumbar musculature is extremely important. ${ }^{2}$ Especially, the multifidus muscles seem to play a key role.

With cross-sectional imaging, atrophy of the multifidus muscles in chronic low back pain can be found, and targeted exercise showed an improvement in back pain. ${ }^{2}$ The multifidus muscles as well as the facet joints are innervated by the medial branch of the dorsal ramus of the spinal nerve. ${ }^{3}$ For diagnosis and treatment of facet joint syndrome, the medial branch is a common target for infiltration with local anesthetics, steroids, or radio-frequency denervation (medial branch block). The concomitant innervation of the multifidus muscles by the medial branch raises several questions. On one hand, medial branch blocks may not be a specific test of facet jointmediated pain, and part of the pain relief may be due to the relaxation of muscles.

Of greater concern are atrophic changes of the multifidus muscles after radio-frequency neurotomy of the medial branch, which have been shown by Dreyfuss et al. ${ }^{4}$ In this small case series, all 5 patients showed diffuse atrophic changes of the multifidus muscles on crosssectional imaging. Because the patients still had pain relief 12 months after the intervention, the authors concluded that the observed atrophy is of little clinical significance. The diffuse atrophy is in accordance with the findings of $\mathrm{Wu}$ et al, ${ }^{5}$ who described polysegmental atrophic changes of the multifidus muscles after radio-frequency denervation of 1 level by using electromyography. In my opinion, the problem of atrophy after medial branch interventions of the segmental stabilizing musculature has been underestimated, especially because atrophic changes of the multifidus muscles have been linked to postdiskotomy syndrome. ${ }^{6}$

The long-term sequelae of the atrophy of multifidus muscles after radio-frequency denervation of the medial branch are unknown. Given the fact that the median duration of clinical improvement after radio-frequency denervation is between 6 and 12 months, we may help patients in the short term yet make them more susceptible to further episodes of low back pain in the future. I propose a modifica- tion of the infiltration technique that may help to solve the abovementioned problems. According to Bogduk et al, ${ }^{3}$ articular branches arise from the medial branches and run cranially to caudal parts and caudally to cranial parts of each facet joint capsule. With the use of CT, I try to target these nerves by placing the tip of the needle at the superior as well as the inferior part of the joint space of each facet joint. With these target points, the medial branch running toward the multifidus muscles should be protected.

The feasibility of a quite similar approach by using CT fluoroscopy has been shown by Meleka et al. ${ }^{7}$ In this study, the immediate postintervetional pain relief of pericapsular infiltrations was even better than that with conventional fluoroscopically guided medial branch blocks. We believe that this pericapsular approach is more specific and possible deleterious long-term effects on the stabilizing musculature of the spine could be avoided. For diagnostic and therapeutic blocks, intra-articular injections may also be used. However, in a number of patients, these cannot be performed because of prominent osteophytic changes, and rupture of the joint capsule with leakage into the epidural space can occur, reducing the sensitivity of this approach.

The equal efficacy of intra-articular and periarticular infiltrations has been shown by Lilius et al. ${ }^{8}$ Pericapsular injections are easy to perform, and the same target points can be used for diagnostic and therapeutic interventions as well as radio-frequency denervation. I also advocate facet joint interventions as part of a multidisciplinary treatment approach, also including targeted exercise of the paraspinal muscles. Clearly more studies on this important topic are needed.

\section{References}

1. Binder DS, Nampiaparampil DE. The provocative facet joint. Curr Rev Musculoskeletal Med 2009;22:15-24

2. MacDonald DA, Moseley GL, Hodges PW. The lumbar multifidus: does the evidence support clinical beliefs? Man Ther 2006;11:254-63. Epub 2006 May 23

3. Bogduk N, Wilson AS, Tynan W. The human lumbar dorsal rami. J Anat 1982;134:383-97

4. Dreyfuss $P$, Stout A, Aprill C, et al. The significance of multifidus atrophy after successful radiofrequeny neurotomy for low back pain. $P M R$ 2009;1:719-22

5. Wu PB, Date ES, Kingery ES. The lumbar multifidus muscle in polysegmentally innervated. Electromyogr Clin Neurophysiol 2000;40:483-85

6. Zoidl G, Grifka J, Boluki D, et al. Molecular evidence for local denervation of paraspinal muscles in failed-back surgery/postdiscotomy syndrome. Clin Neuropathol 2003;22:71-77

7. Meleka S, Patra A, Minkoff E, et al. Value of CT fluoroscopy for lumbar facet blocks. AJNR Am J Neuroradiol 2005;26:1001-03

8. Lilius G, Lassonen EM, Myllynen P, et al. Lumbar facet joint syndrome: a randomised clinical trial. J Bone Joint Surg Br 1989;71:681-84

J. Gossner

Department of Clinical Radiology Weende Hospital Göttingen, Germany 